\title{
Effect of using cardiovascular risk scoring in routine risk assessment in primary prevention of cardiovascular disease: an overview of systematic reviews
}

\author{
Krzysztof Studziński ${ }^{1,3}$, Tomasz Tomasik ${ }^{1,3^{*}}$ (D, Janusz Krzysztoń ${ }^{1,3}$, Jacek Jóźwiak ${ }^{2,3}$ and Adam Windak ${ }^{1,3}$
}

\begin{abstract}
Background: Our objectives were to critically appraise and summarise the current evidence for the effectiveness of using cardiovascular disease (CVD) risk scoring (total risk assessment - TRA) in routine risk assessment in primary prevention of CVD compared with standard care with regards to patients outcomes, clinical risk factor levels, medication prescribing, and adverse effects.

Methods: We carried out an overview of existing systematic reviews (SRs). Presentation of the results aligned guidelines from the PRISMA statement. The data is presented as a narrative synthesis. We searched MEDLINE (Ovid), EMBASE, CENTRAL and SCOPUS databases from January 1990 to March 2017, reviewed the reference lists of all included SRs and searched for ongoing SRs in PROSPERO database. We encompassed SRs and meta-analyses which took into account RCTs, quasi-RCTs, and observational studies investigating the effect of using CVD risk scoring. Only studies performed in a primary care setting, with adult participants free of clinical CVD were eligible. Intervention was CVD risk assessment with use of the total CVD risk scoring compared with standard care with no use of TRA .
\end{abstract}

Results: We identified 2157 records, we then recognised and analysed 10 relevant SRs. One SR reported statistically insignificant reduction of CVD death, when using TRA, the second SR presented meta-analysis which reported no effect on fatal and non-fatal CV events compared with conventional care (5.4\% vs 5.3\%; RR 1.01, 95\% Cl 0.95 to $1.08 ; 1^{2}=25 \%$ ). Three SRs have shown that using TRA causes no adverse events. The impact of TRA on global CVD risk as well as individual risk factors is ambiguous, but a tendency towards slight reduction of blood pressure, total cholesterol and smoking levels, especially in high risk patient groups was observed. TRA had no influence on lifestyle behaviour.

Conclusions: There is limited evidence, of low overall quality, suggesting a possible lack of effectiveness of TRA in reducing CVD events and mortality, as well as a clinically insignificant influence on individual risk factor levels. Using TRA does not cause harm to patients.

Trial registration: Systematic review protocol was registered with the International PROSPERO database - registration number CRD42016046898.

Keywords: Cardiovascular diseases, Primary prevention, Risk assessment, Cardiovascular system, Risk factors

* Correspondence: mmtomasi@cyf-kr.edu.pl

${ }^{1}$ Department of Family Medicine, Chair of Internal Medicine and

Gerontology, Jagiellonian University Medical College, 4 Bochenska Street,

31-061 Krakow, Poland

${ }^{3}$ College of Family Physicians in Poland, Warszawa, Poland

Full list of author information is available at the end of the article

(c) The Author(s). 2019 Open Access This article is distributed under the terms of the Creative Commons Attribution 4.0 International License (http://creativecommons.org/licenses/by/4.0/) which permits unrestricted use, distribution, and reproduction in any medium, provided you give appropriate credit to the original author(s) and the source, provide a link to the Creative Commons license, and indicate if changes were made. The Creative Commons Public Domain Dedication waiver (http://creativecommons.org/publicdomain/zero/1.0/) applies to the data made available in this article, unless otherwise stated. 


\section{Introduction}

Cardiovascular disease (CVD) is presently the leading cause of mortality, morbidity and disability worldwide $[1,2]$. Well-established, modifiable CVD risk factors include: elevated blood pressure, hypercholesterolaemia, diabetes, lack of physical activity, obesity, inappropriate diet, and smoking [3, 4].

In 1961, Kannel et al. [5] used the term "factors of risk" for the first time and proved that hypertension, hypercholesterolaemia and other risk factors precede the development of coronary heart disease [CHD] in humans.

Understanding CVD risk factors makes prevention possible. Risk factor modification can reduce the number of premature deaths as well as the number of other clinical events, both in people without established CVD (primary prevention) and people with confirmed CVD (secondary prevention) [6].

Following Kannel's work, a new concept of the absolute/global/total CVD risk has become widely adopted. By definition, absolute CVD risk is the actual risk of developing the disease within a defined population in a defined period of time (typically 5 or 10 years) [7]. The absolute CVD risk is calculated by using a combination of major risk factors. Risk score algorithms incorporating multiple risk factors are used to calculate absolute risk for an individual patient [8].

The number of risk scores developed in European countries, USA and other parts of the world have grown rapidly. In 2016, Damen et al. [9] identified 363 prognostic models or risk-scoring methods with potential use in targeting primary prevention of CVD. Contemporarily, the most well-known and widely used are: Systematic COronary Risk Evaluation (SCORE) algorithm [10], QRISK2 [11], the World Health Organization (WHO) risk score, and the American College of Cardiology/ American Heart Association (ACC/AHA) 2013 Pooled Cohort risk equations [12].

Presently, major clinical practice guidelines (CCS [13], ESC/ESH [6], ACC/AHA [14], JBS3 [15]) recommend assessing risk of $\mathrm{CVD}$ using the absolute/global/total CVD risk scores. It is stated that the use of CVD risk scores increases the accuracy of prediction of CVD events and guides management decisions in primary prevention.

The effectiveness of using absolute/global/total CVD risk scores called also total risk assessment (TRA) has been assessed by numerous randomised control trials (RCTs), published over the last two decades. The research community also tried to summarise existing evidence in this field, which resulted in the publication of a few systematic reviews (SRs) [16-19]. However, despite numerous studies, there are still some unsolved issues. One of them is whether using TRA is clinically effective when important outcomes for patients are taken into account (e.g. mortality, CV events). Even when TRA appropriately predicts $\mathrm{CV}$ events, it does not mean that beneficial clinical effects will occur. Furthermore, there is a lack of data about the potential adverse effects of TRA. The absence of information does not mean that this procedure is completely safe.

Summing up, the effectiveness of using TRA in clinical practice is still poorly understood. Although it may be beneficial and improve health (e.g. identifying high-risk individuals who will most likely benefit from risk factor management), it may also be harmful (e.g. undertreatment of the youth, overtreatment of the elderly) and lead to misuse of resources (e.g. time and cost of laboratory tests).

\section{Objectives}

This study has the following objectives: (1) to critically apprise and summarise the best current evidence for the effectiveness of using TRA in routine risk assessment in primary prevention of CVD compared with standard care; (2) to assess whether use of a particular risk score followed by structured or unstructured intervention is more effective than any other risk score in improving patient outcomes and (3) to discuss how our findings can be used to guide clinicians and policymakers, and provide a guideline for future authors.

\section{Methods}

\section{Protocol and registration}

Our systematic review protocol was registered with the International Prospective Register of Systematic Reviews (PROSPERO) on 01 September 2016 (registration number CRD42016046898). The full version of the protocol was later published in the BMJ Open [20]. Any deviations from this plan, which were minor, are described in Additional file 1.

\section{Eligibility criteria}

Studies were selected according to the prespecified criteria defined below. Those criteria were also mentioned in detail in the full version of the protocol of our study published in the BMJ Open [20].

\section{Study design}

We performed an overview of SRs and meta-analyses which took into account RCTs, quasi-RCTs, and observational studies investigating the effect of using CVD risk scoring in routine risk assessment in primary prevention of CVD.

\section{Participants}

Only studies with adult participants (19 years of age and over) and free of clinical CVD, were eligible. Studied patients may have had different CVD risk factors or other diseases including diabetes and chronic kidney disease. 


\section{Intervention}

CVD risk assessment with use of total risk assessment (TRA), performed by physicians or other healthcare professionals.

\section{Comparator}

Standard care with no use of the global CVD risk scoring provided by a physician or healthcare professional.

\section{Outcomes}

Primary outcomes (1) CVD death, (2) Fatal and nonfatal cardiovascular events, (3) Adverse events (any physical, psychological or social events).

Secondary outcomes (1) All-cause mortality, (2) Change in predicted global CVD risk, (3) Change in patient CVD risk factors - change in blood pressure, cholesterol level, smoking, exercise, diet, alcohol consumption and obesity, (4) Prescription of risk-reducing drugs according to prevailing guidelines (aspirin, antihypertensives, lipid-lowering drugs), (5) Pharmacotherapy without or against current clinical guideline recommendations.

Adverse events mentioned as primary outcomes could be as follows: (1) physical - e.g. hypertension or dyslipidaemia complications in young patients who were excluded from pharmacotherapy due to low CVD risk score; (2) psychological - e.g. anxiety, depression, stress caused by diagnosis and being labelled as "chronically ill"; (3) social - e.g. cost and additional time spent on unnecessary consultations, role changing in family or society.

\section{Setting}

Only studies performed in an outpatient setting were eligible since TRA guides management decision in primary prevention in patients without known CVD.

\section{Search strategy}

We searched MEDLINE (Ovid), EMBASE, CENTRAL (Cochrane Central Register of Controlled Trials) and SCOPUS databases from January 1990 to March 2017. All databases from 1990 onwards were scanned because only a very small number of systematic reviews had been conducted before that time $[21,22]$. The search was limited to English language literature. It was supplemented by a search for unpublished, ongoing, or recently completed systematic reviews in PROSPERO database. In addition, we reviewed the reference lists of all included systematic reviews. Our detailed search strategy for MEDLINE is presented in Additional file 2.

\section{Data management}

All identified records were uploaded or manually entered into Mendeley v1.17.6 (Elsevier). After duplicate removal, titles and abstracts from the searches were independently screened by two authors. Full-text articles were retrieved for all potentially includable SRs. Any disagreements were resolved through discussion. In case of lack of consensus, a third author was arbitrated.

The methodology for data extraction and analysis was based on guidance from Preferred Reporting Items for Systematic Reviews and Meta-Analyses (PRISMA) statement [23] and the Cochrane Handbook of Systematic Reviews of Interventions [24]. The PRISMA checklist is presented in an Additional file 3. Two authors independently extracted outcome data from each included SR using a predefined data extraction form. Disagreements during the data extraction process were resolved through discussion or arbitrarily by a third author.

We have extracted the following information: (1) administrative and bibliographic data; (2) the characteristics of each review; (3) methodological details and results of meta-bias assessments (if conducted); (4) reported limitations of each review; (5) results and conclusions of the review.

If meta-analysis was presented in one of the included SRs, we extracted both its results and all relevant methodological aspects (e.g. types and unity of data, effect measured, heterogeneity, sensitivity analysis). As we specified in our overview protocol [20], we did not conduct a meta-analysis of meta-analyses, mainly because of the risk of introducing bias. According to Smith et al. [25] and Pieper et al. [26], such undesired bias can be easily incorporated (due to giving excessive statistical power to primary studies included in more than one systematic review) when performing meta-analysis in review of systematic reviews. Additionally, the same authors highlight that there is no well-established quantification method in this field.

Additionally, we looked at the following information: (1) outcomes reported in a particular SR which were recognised by the authors as evidence of the effectiveness of TRA, (2) reported effect of TRA in different populations, (3) reported effect of different TRA in the same population.

\section{Risk of bias in included systematic reviews}

Two reviewers independently appraised risk of bias of the included systematic reviews using a validated assessment of multiple systematic reviews (AMSTAR) checklist, which is the most commonly employed method to assess the quality of systematic reviews included in overviews [27]. We scored each systematic review with a maximum of 11 points. Disagreements were resolved through discussion or arbitrarily by a third author. Each 
systematic review was assigned to one of three quality levels (0-3 points - low quality, 4-7 points - medium quality and $8-11$ points - high quality) $[28,29]$.

\section{Risk of bias across systematic reviews}

To assess selective outcome reporting within systematic reviews, we compared those which were planned to be assessed in the systematic review protocols (or, if unavailable, in the methods section of the published report of SR) and other outcomes reported in the results section of the published report of SR. To minimise publication bias, we identified all relevant ongoing SRs by searching the PROSPERO database. To assess the degree of overlap in the inclusion of primary studies between systematic reviews, the citation matrix was generated by one reviewer and checked by a second for accuracy (Additional file 4). The degree of overlap was calculated with use of the corrected cover area (CCA) in our citation matrix by an experienced statistician [26].

\section{Results}

After duplicates were removed, the electronic search identified 2045 papers. One hundred twelve records from other sources were added (104 records of ongoing or recently completed SRs from PROSPERO database, 7 papers found in the reference sections of known SRs and one received due to contact with the authors). 2157 records were independently screened by two authors. Fifteen full-text articles were screened for eligibility, of which 4 were not related to the effectiveness of TRA [30-33], 1 was not SR [34], and the remaining 10 SRs included 66 unique primary studies and were included (see Additional file 4). Flow diagram is presented at Fig. 1.

\section{Summary of included systematic reviews}

Characteristics of included systematic reviews are presented in Table 1.

The review performed by Brindle et al. [16] focused both on the accuracy and impact of CVD risk assessment in primary prevention. The authors performed comprehensive searches across 6 databases. The majority of included studies focused on CVD risk assessment accuracy. The authors identified 4 RCTs assessing effectiveness of using CVD risk score. As the quality of evidence was unclear, the authors concluded that it was insufficient to support the claim that CVD risk assessment performed by clinicians improves health outcomes.

Sheridan and Crespo [17] focused on the benefits and harms of physicians' knowledge of CVD risk scoring. The authors searched one database (MEDLINE) and identified 11 primary studies. They concluded that "physicians' knowledge of global CHD risk scores may translate into modestly increased prescribing of cardiovascular drugs and modest short-term reductions in CHD risk factors without clinical harm".

The SR by Sheridan et al. [18] aimed at evaluating the effectiveness of presenting CVD risk information to patients as a primary intervention versus part of a multipart intervention. The researchers studied 4 databases and considered studies of any quantitative experimental design. They identified 18 primary studies of good or fair quality. Results suggested that providing CVD risk information to the patients may "improve accuracy of risk perception and may increase intent to initiate CHD prevention". However, the effect on more long-term outcomes was not clear.

Waldron et al. [35] tried to assess the effectiveness of using different methods of communicating CVD risk and the impact of these methods on patient-related outcomes. The authors performed comprehensive searches across 6 databases. However, only 4 out of the 15 quantitative studies included assessed individuals' actual risk. The majority of these studies were analogue studies using hypothetical risk profiles. The authors concluded that there were not many well-designed studies focusing on CVD risk communication.

In their review, Van Dieren et al. [36] aimed to identify all papers presenting CVD prediction models developed in either diabetic or general patient populations that included diabetes as a predictor. The review also identified studies examining the impact of applying a prediction model in clinical practice. They found 3 studies of this type, all of which utilised the Framingham prediction model. Results suggested that it is still unknown whether the use of these models indeed changes the treatment of patients with diabetes mellitus or whether it reduces the number of cardiovascular complications.

Willis et al. [19] aimed to evaluate the effectiveness of using validated CVD risk scores to identify individuals at highest risk followed by intervention as a means of reducing CVD risk or mortality. They included 5 full texts in their review. Two trials reported a reduction in CVD mortality. However one of them showed a lack of statistical significance, and in the second, the $p$ value was not provided. The authors concluded that "evidence suggests that lifestyle interventions aimed at the primary prevention of CVD that use validated risk scores to recruit high risk individuals show potential for lowering CVD mortality and the incidence of cardiovascular events".

Usher-Smith et al. [37] searched for interventional studies which involved providing a CVD risk assessment to patients or their providers. Final analysis was based on 17 primary studies. Results suggested that providing patients with risk information improves the accuracy of perceived risk without increasing anxiety or decreasing quality of life. Additionally, it leads to a small reduction in cholesterol and blood pressure despite a lack of 


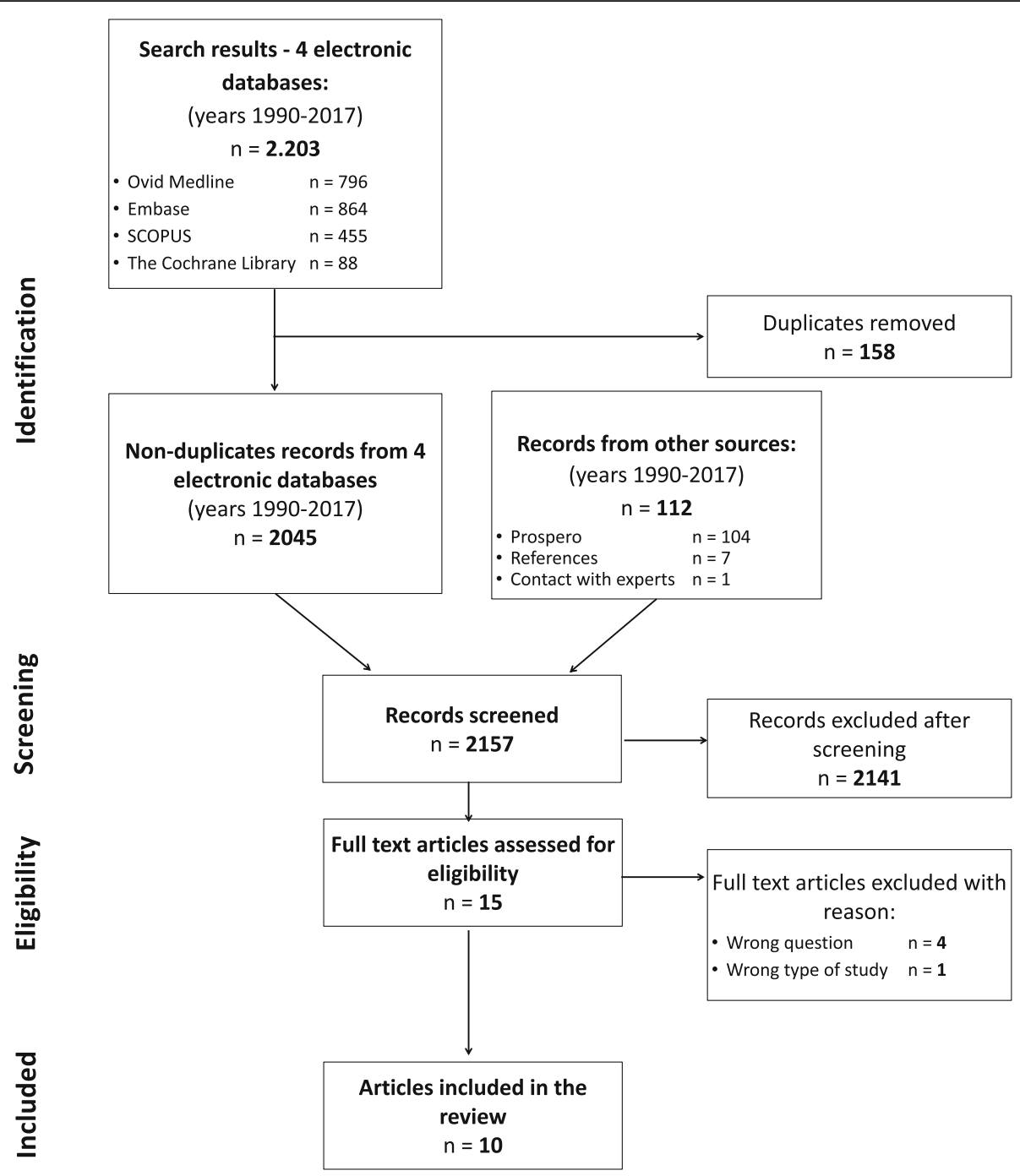

Fig. 1 Flow diagram

evidence that this reduction is due to lifestyle changes. Moreover, providing risk information increases the frequency of prescribing lipid-lowering and blood pressure medications.

Tomasik et al. [38] focused only on the effect of using the specifically European total CVD risk estimation from SCORE. A comprehensive search did not reveal any study that compared important clinical outcomes (death, major events, adverse events) between groups that used the SCORE model and those who did not. The authors concluded that a demonstration of the benefits of using SCORE is still lacking and that current use of this tool as a preventive strategy is not supported by evidence.

The Cochrane review by Karmali et al. [39] focused on the effect of physicians, other healthcare professionals or the health system providing a multivariable CVD risk score. They identified 41 RCTs having multiple limitations, substantial heterogeneity and high or unclear risk of bias. Results suggested that the use of CVD risk scores has little or no effect on CVD events compared with standard care, though to a small extent it may reduce risk factor level (systolic BP, total cholesterol), multivariable CVD risk, or adverse events. It may also increase the use of antihypertensive and lipid-lowering pharmacotherapy.

The Collins et al. [40] review of systematic reviews summarises results from 6 SRs on the impact of global CVD risk assessment in primary prevention. No identified review reported results such as mortality or CVD morbidity. The authors also undertook ad hoc meta-analysis and extracted data from 16 primary studies of the identified publications. They showed small and possibly clinically insignificant reductions in systolic BP, cholesterol level and smoking cessation. The quality of evidence was low or very low (GRADE score). 


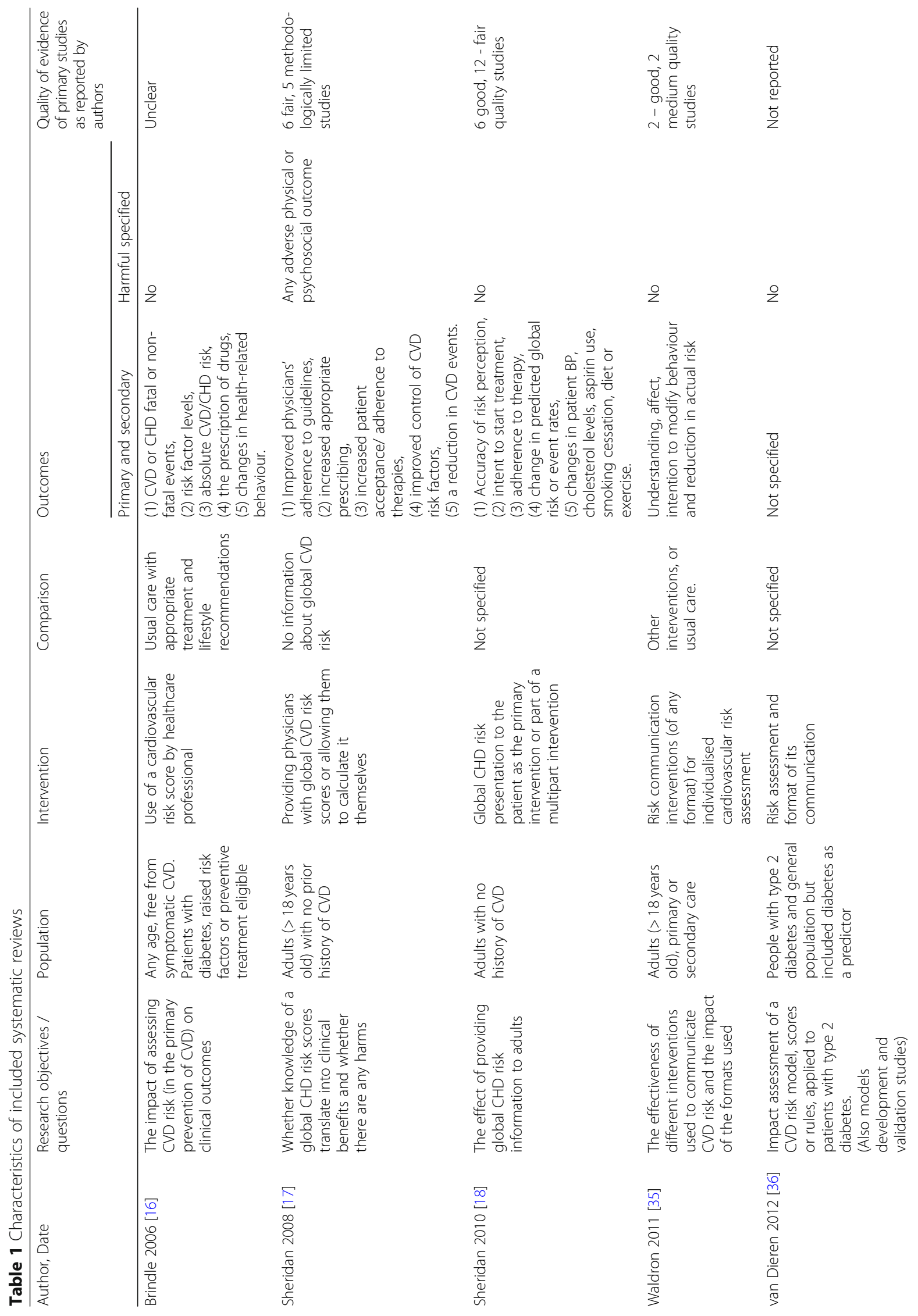




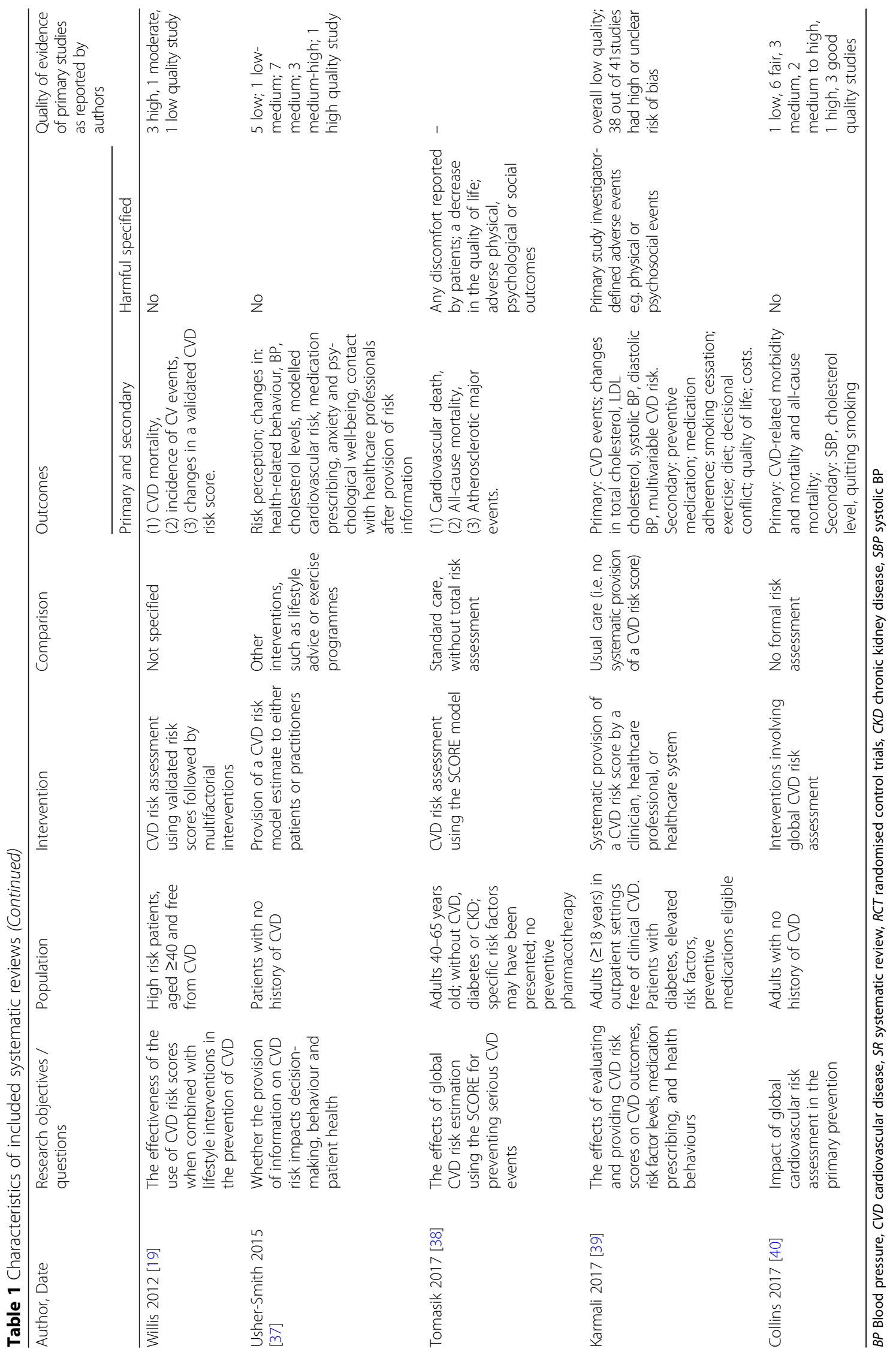


The general conclusions about the included SRs is that there is currently little evidence that providing TRA has a significant impact on important patient outcomes.

\section{Biases in included systematic reviews and across systematic reviews}

AMSTAR ratings ranged from 3/11 to $10 / 11$ (mean 6.1/ 11); no SRs were excluded based on quality (see Fig. 2). Ten eligible SRs included 66 unique primary studies. Thirty five publications were encompassed in only one SR and 31 in more than one. The measure of overlap by corrected covered area (CCA) was $9 \%$, indicating a moderate overlap [26]. Details of calculations and the list of primary studies are presented in Additional file 4.

In the majority of the SRs, the authors presented the same outcomes in the results sections that they had planned to assess in their initial protocols or in the methodology section of published reports. Collins et al. [40] deviated from their registered protocol, which they reported in Additional file 1, published online. They had originally planned to explore many different outcomes, but instead focused on what they deemed to be the most important ones: CVD-related morbidity and mortality as primary outcomes and systolic blood pressure, cholesterol, and smoking as secondary outcomes. Willis et al. [19] additionally reported cardiovascular disease risk factors which were not in the original scope of their work.

\section{Effects of interventions Primary outcomes}

CVD death and fatal and non-fatal CVD event The SR by Willis et al. [19] included two original studies reporting changes in coronary [CHD] death $[41,42]$. In one of the studies [41] the authors reported a lowering of CVD deaths of $4.7 \%$ without indicating if the difference is statistically significant. In another study [42], the reduction of $\mathrm{CHD}$ deaths of $7.4 \%$ was not statistically significant $(p>0.05)$. These two original studies were not mentioned or included in any other SR. Both studies were published in the 1980's, when strategies for CVD risk reduction were far more limited. Additionally, these trials were really designed with the intent of examining the effectiveness of a high intensity intervention geared at risk factor modification, rather than the use of risk scores. Both studies only included men.

Karmali et al. [39] presented a meta-analysis of three studies on the effect of providing TRA on fatal and

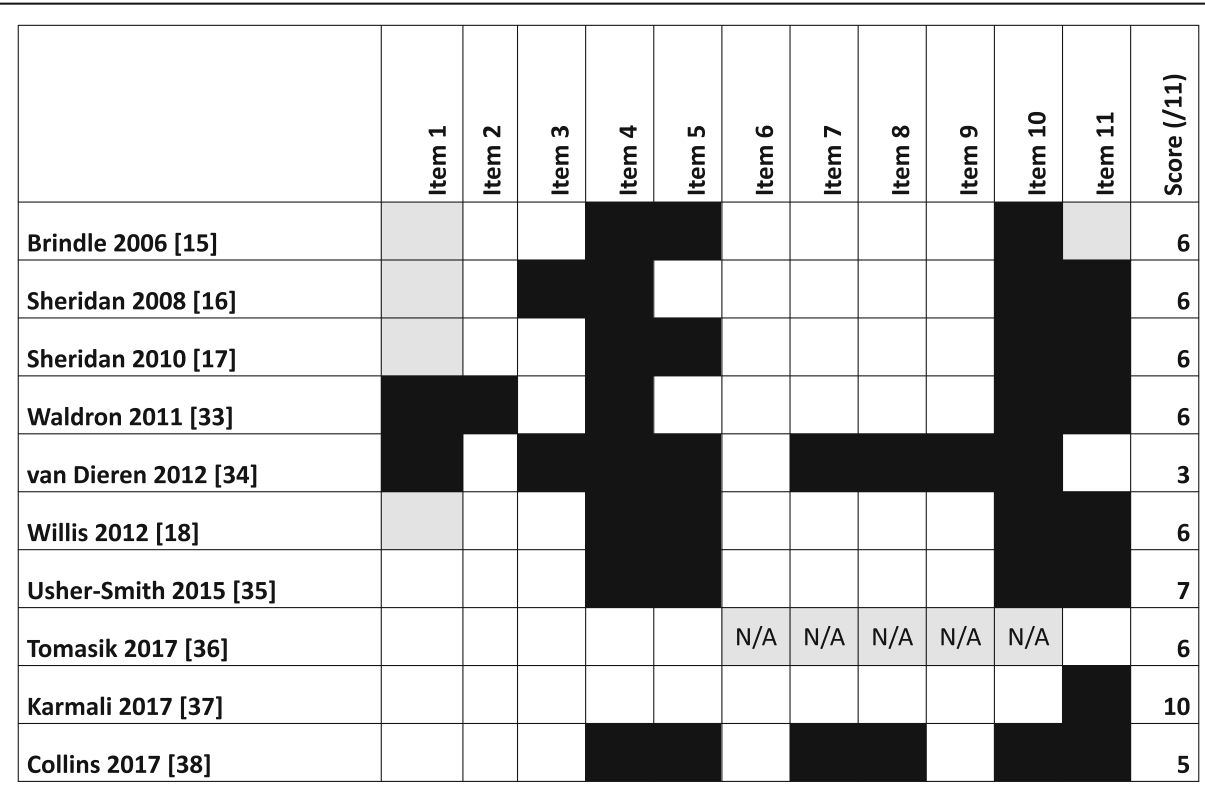

Item 1: Was an 'a priori' design provided?; Item 2: Was there duplicate study selection and data extraction?; Item 3: Was a comprehensive literature search performed?; Item 4: Was the status of publication used as an inclusion criterion?; Item 5: Was a list of studies provided?; Item 6: Were the characteristics of the included studies provided?; Item 7: Was the scientific quality of the included studies assessed and documented?; Item 8: Was the scientific quality of the included studies used appropriately in formulating conclusions?; Item 9: Were the methods used to combine the findings of studies appropriate?; Item 10: Was the likelihood of publication bias assessed?; Item 11: Was the conflict of interest included?.

Fig. 2 Assessment of the methodological quality of the included systematic reviews (AMSTAR). Legend: White = Yes; Black= No; Grey=can't answer/unclear; N/A = not applicable 
non-fatal $\mathrm{CV}$ events. It reported no effect compared with conventional care $(5.4 \%$ vs $5.3 \%$; risk ratio [RR] $1.01,95 \%$ confidence interval $[\mathrm{CI}] 0.95$ to $1.08 ; \mathrm{I}^{2}=25 \%$ ). Included in meta-analysis studies had low quality with high or unclear risk of bias. Furthermore, 2 of these studies were likely underpowered for assessment of CVD events due to limited recruitments of participants over the age of 50 years and low rates of CVD events. The third study included a cohort of patients with HIV, which is not generalizable.

Adverse events Authors of three SRs [17, 37, 39] were looking for possible adverse events of CVD risk assessment. These SRs showed no difference in the presence of psychological symptoms in groups with or without TRA. Usher-Smith et al. [37] also showed a small improvement in psychological well-being (measured with GHQ-28 scores) and level of anxiety in high CVD risk patients after TRA.

One SR [39] reported low-quality evidence suggesting that providing TRA may reduce the presence of adverse physical events (1.9\% vs $2.7 \%$; RR $0.72,95 \%$ CI 0.49 to 1.04; $\mathrm{I}^{2}=0 \%$ ).

The detailed results of all primary outcomes are presented in Table 2.

\section{Secondary outcomes}

All-cause mortality None of the included SRs reported changes in all-cause mortality.

Change in predicted global CVD risk and CVD risk factors. Four SRs reported reduction of predicted global CVD risk after providing TRA [17, 19, 37, 39]. One SR [18] showed mixed results and another SR [16] showed no difference. Seven SRs showed a tendency towards a small reduction of BP [16-19, 37, 39, 40]. The authors of eight SRs assessed possible changes in cholesterol level. They showed mixed results with the tendency towards reduction of TC [16-19, 35, 37, 39, 40]. There was no change in reported obesity level $[17,37]$, diet used [16, 37,39 ] or alcohol consumption [37], but a slight reduction in smoking was noted $[18,19,37,39,40]$.

Details are presented in Table 3.

Prescription of risk-reducing drugs according and against guidelines Five SRs [16, 17, 36, 37, 39] reported the impact of TRA on prescription of risk-reducing drugs. The results were mixed, but in all they suggest that TRA use may increase or have no effect on prescription of aspirin, anti-hypertensive and lipid-lowering drugs in all CVD risk groups. They also suggest that prescription of anti-hypertensive and lipid-lowering drugs in high CVD risk groups is increased.
One SR [39] reported the effect of providing TRA to patients on prescription of drugs against guidelines.

Detailed data is presented in Table 4.

We were unable to find any studies comparing the use of different risk models followed by structured or unstructured intervention showing that one is more effective than another.

The majority of SRs $(n=8)$ recommend that further effectiveness studies are needed. Five SRs propose high quality RCTs. One of these SRs proposes a cluster RCT with CVD mortality and morbidity data as primary outcomes. Two SRs propose trials with surrogate outcomes (CVD risk factor levels), which may be more feasible due to a smaller population and less time needed.

\section{Discussion}

\section{Main findings}

We have identified and analysed 10 SRs which were published between 2006 and 2017. Two of them reported the impact of performing TRA on mortality, fatal and non-fatal CV events. One medium quality SR [19] revealed 2 primary studies reporting reduction of CVD death, but it was statistically insignificant or significance was not reported in the original study. Other high quality SR [39] presented meta-analysis of 3 primary studies which showed no significant differences concerning fatal and non-fatal CV events. Primary studies included in both SRs had important limitations.

Three SRs [17, 37, 39] have analysed the impact of performing TRA on adverse events. It was shown that using TRA causes no difference in presence of psychological or physical events.

The impact of TRA on global CVD risk and individual risk factors is ambiguous, but a tendency towards slight reduction of $\mathrm{BP}, \mathrm{TC}$ and smoking levels, especially in high risk patients group, was observed. TRA had no influence on lifestyle behaviour.

\section{Strengths and limitations}

To the best of our knowledge this is the largest overview of SRs in this area. We included 10 SRs while a previous overview published in March 2017 covered six [40]. Our overview of SRs was conducted strictly according to the protocol, which was registered with the PROSPERO database, and later published in the BMJ Open [20]. Other strengths are a robust search strategy prepared by an experienced librarian, and broad inclusion criteria.

There are some obvious limitations to our overview of SRs. We did not review the quality of each study included in the SRs, nor perform an independent analysis of data (which is standard procedure when performing overview of SRs). The electronic search strategy was limited to 4 main international bibliographic databases and English language literature only. Grey literature for 


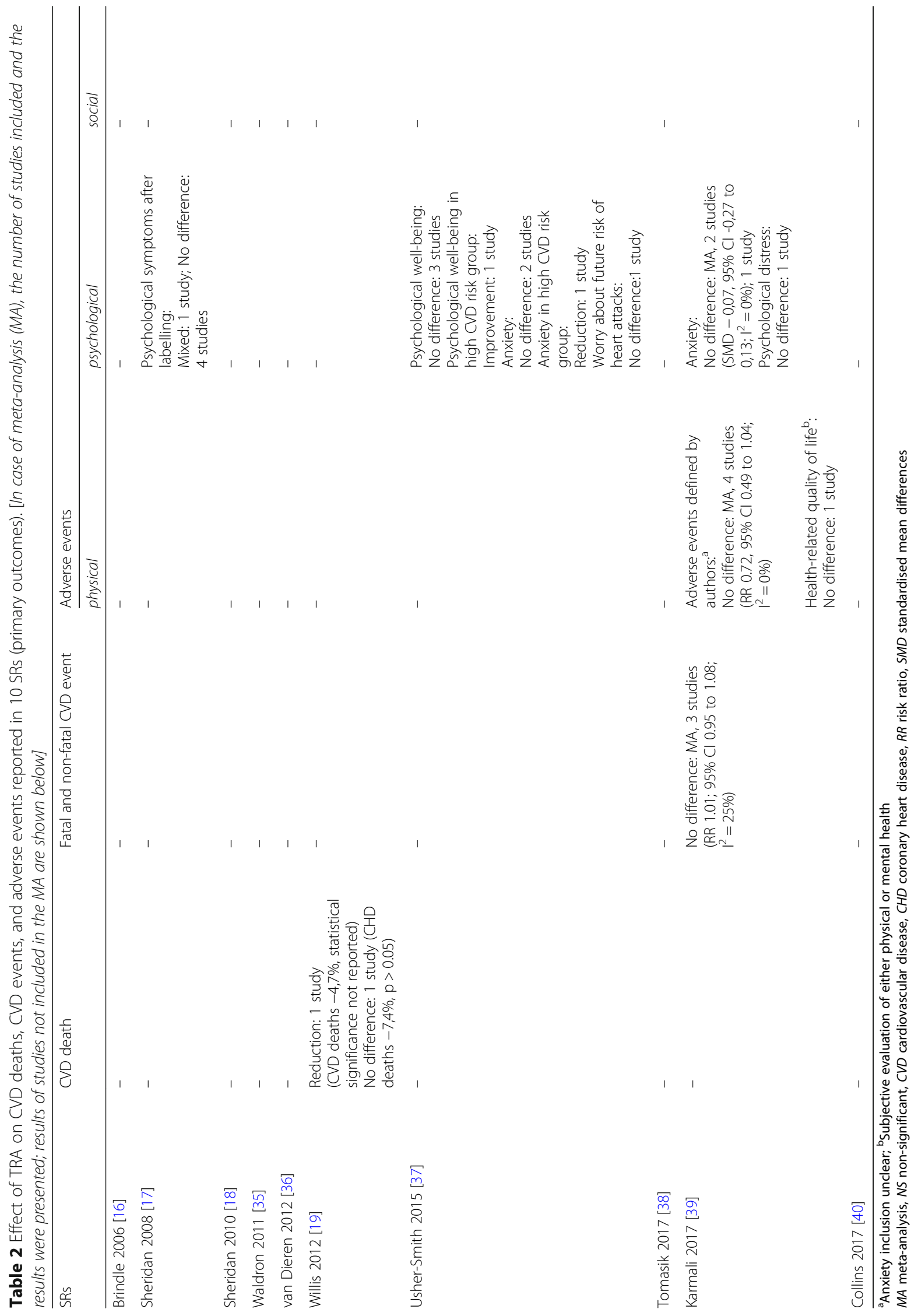




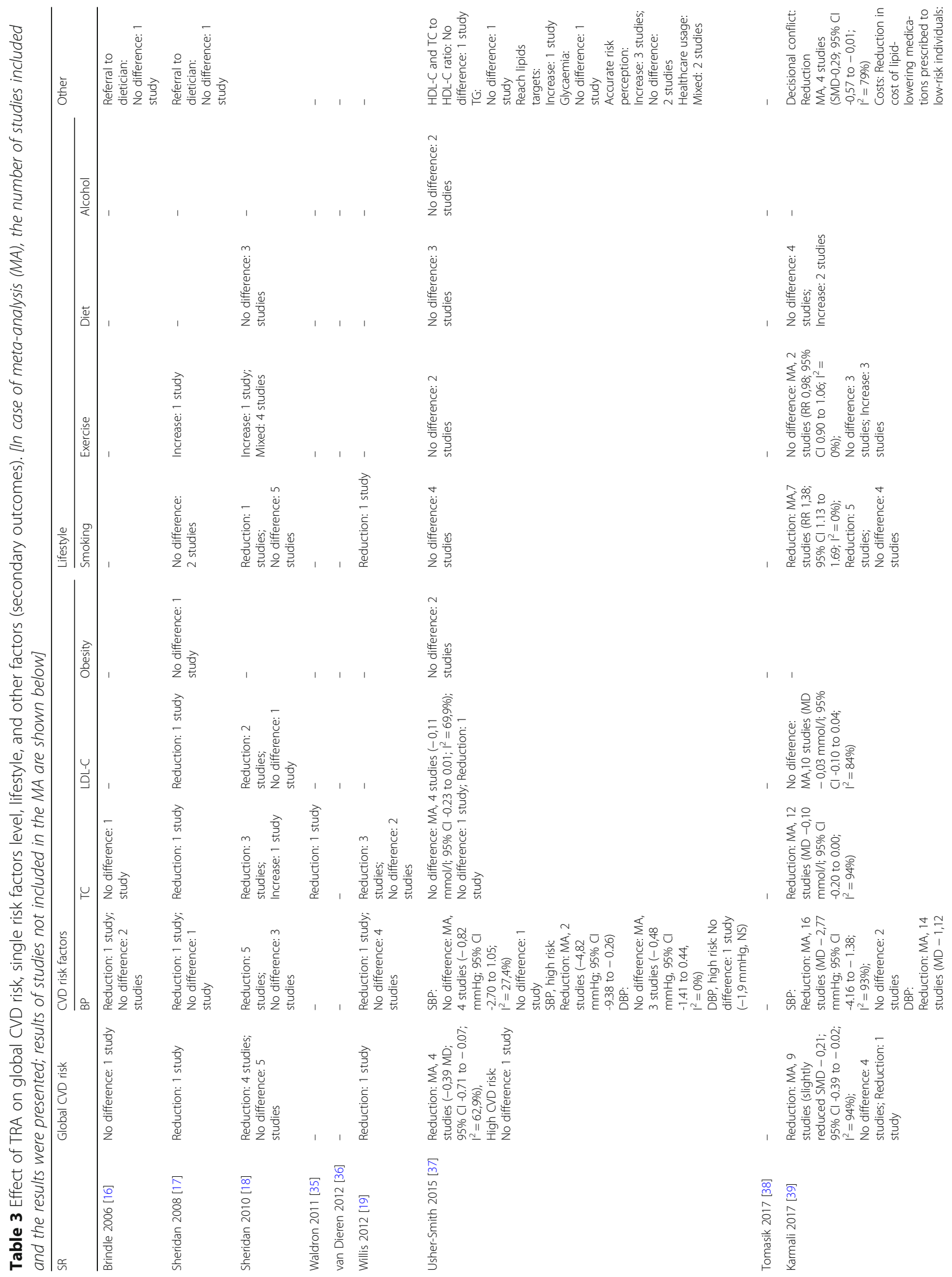




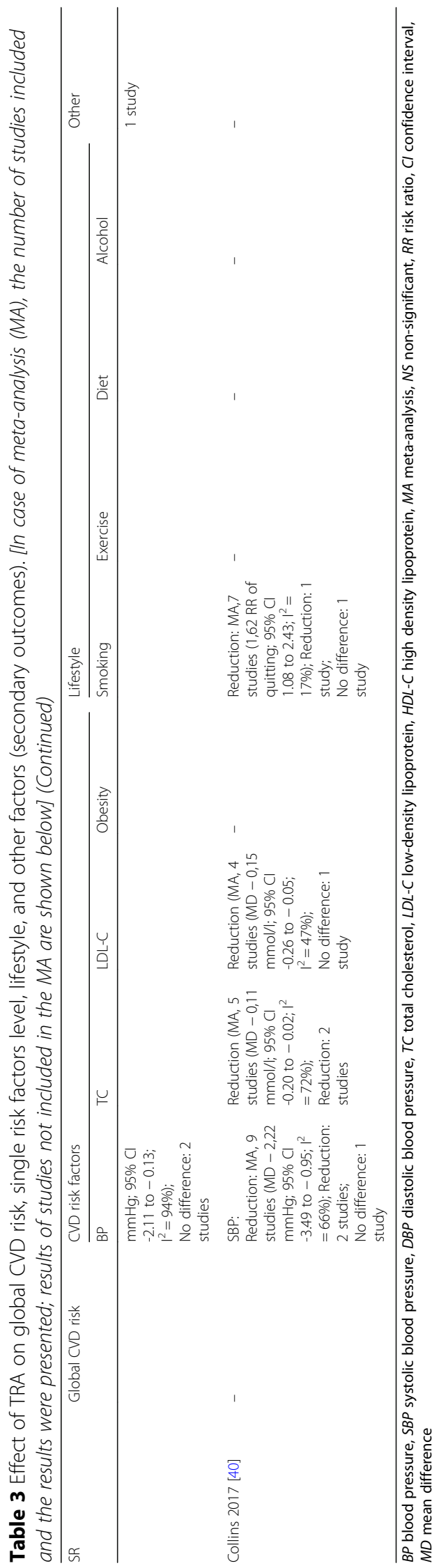




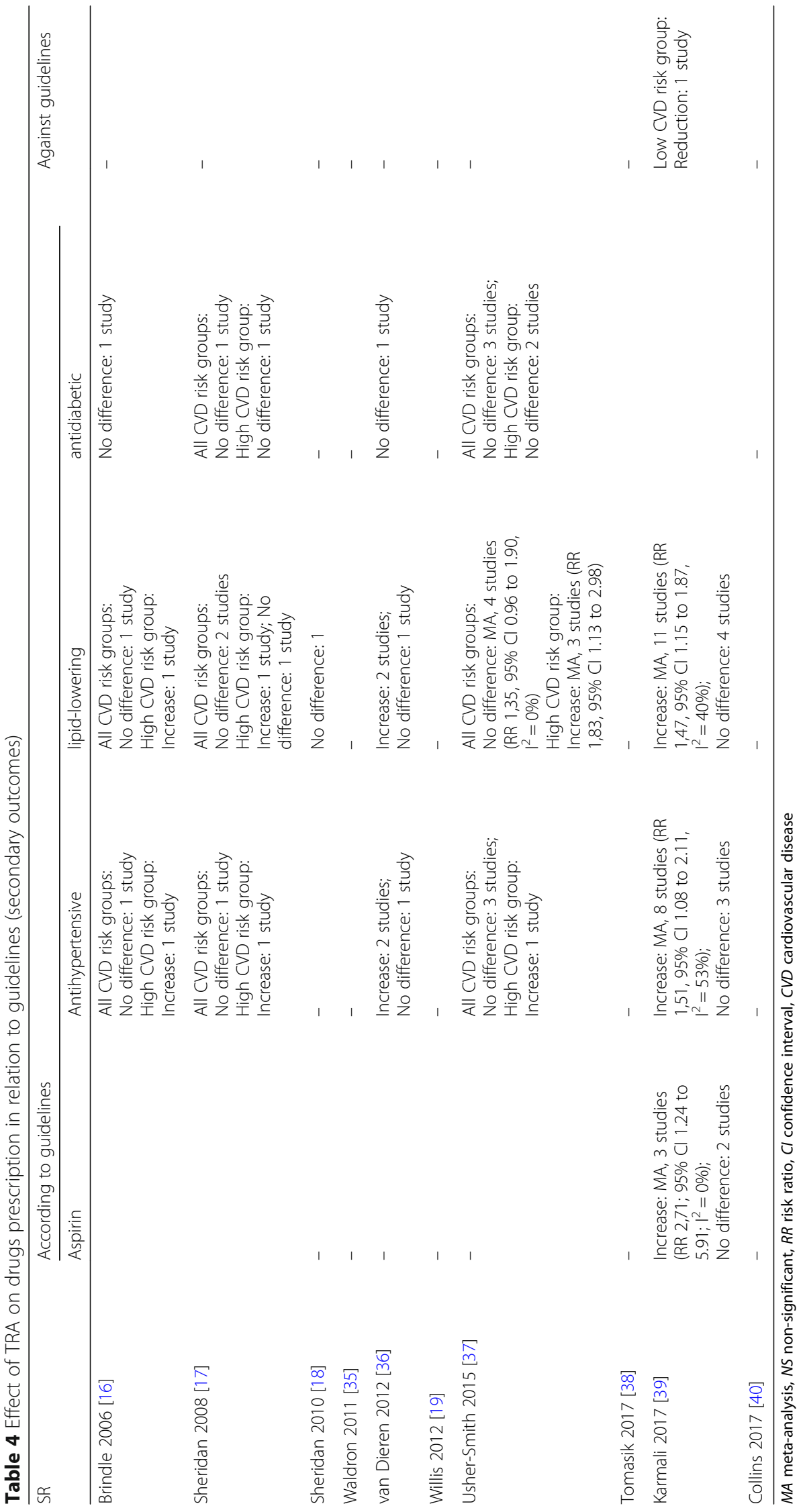


unpublished papers was not studied, however a search for ongoing or recently completed systematic reviews in the PROSPERO database was performed. In addition, we also manually reviewed the reference lists of all included SRs.

\section{Comparison with other studies and opinions}

Collins et al. [40] completed searches for their overview of SR in October 2016. They did not find SRs concerning their primary outcomes (CVD mortality and morbidity and all-cause mortality). The conclusions of that overview differ from ours and the authors stated that "there is currently no evidence that the prospective use of global cardiovascular risk assessment translates to reductions in CVD morbidity or mortality". We hold the view that there is a limited evidence for the lack of these reductions.

Results of our overview of SRs can be also compared with evidence constituting the basis for recommendations within national and international guidelines. Major clinical practice guidelines (CCS [13], ESC/ESH [6], ACC/AHA [14], JBS3 [15], NICE [43]) recommend assessing risk of CVD using TRA. It is stated that use of these risk scores increases the accuracy of prediction of CVD events and guides management decisions in primary prevention. Some guidelines indicate that there is no proof for the effectiveness of TRA [6]. Our research has shown the contrary - there is a limited evidence, of low overall quality, for a lack of effectiveness of TRA on CVD events.

There are publications indicating, similar to our study, that some primary prevention interventions may not be effective. The Cochrane review for instance concluded that general health checks performed in healthy individuals do not reduce morbidity or mortality, nor in overall cardiovascular or cancer causes. [32] The Danish Inter99 randomised trial revealed that systematic screening for risk factors of ischaemic heart disease, followed by individually tailored intervention had no effect after 10 years and did not reduce incidence of stroke, ischaemic heart disease, as well as mortality rate [44]. On the other hand Si S. et al. showed that health checks are associated with statistically significant, though clinically small, improvements in surrogate outcomes, especially in high risk individuals [33].

\section{Interpretation of results}

The readers of our overview should take into consideration that the results concerning the effectiveness of TRA comes not from primary studies but are strictly limited to results published in SRs and meta-analyses. Data derived from these types of studies are the most useful evidence in clinical practice [6]. However, our analysis shows a diversity in the quality of included SRs.
The majority of them are, according to AMSTAR, of medium quality. We also present the quality of evidence of primary studies as assessed by authors of the included SRs. Most of them reported a low quality of primary studies. In accordance with the authors of these SRs, we agree that conducting further research is still necessary, in order to obtain more persuasive evidence for TRA effectiveness in primary prevention.

There are many reasons why TRA may not be effective and they are described in the literature. Firstly, false positive results of TRA may cause medical treatment which is unnecessary and brings risk of side effects. Secondly, false negative results may cause the lack of appropriate treatment [6]. Thirdly, only a small number of risk variables are included in most of the models (they do not include e.g. family history, TG level, or obesity). Risk categories can be arbitrary (e.g. low, medium, high). In addition, mortality and morbidity rates are declining in most European countries but prediction models are based on historic data and may not apply to the population to be assessed. Moreover, a small change in one of the individual risk factors (e.g. blood pressure) from 1 day to another can markedly change patients' estimated CVD risk. Finally many physicians identify their patients' CVD risk factors and launch appropriate treatment without the necessity of TRA [45].

\section{Implications}

Even though TRA is recommended by major clinical practice guidelines, medical professionals should be aware that current, limited and of low quality, evidence from SRs indicate a possible lack of its clinical effectiveness. Using TRA tools will not harm the patients. There are no grounds to conclude that, in clinical practice, CVD risk assessment based on other than TRA methods, e.g. only on one particular risk factor or counting the number of risk factors instead of using TRA, is inappropriate. However, there are several other potential benefits associated with the use of TRA which are considered in the current guidelines.

Guideline authors should consider re-evaluating the class of recommendations. In the 2016 European Guidelines on CVD prevention [6], the recommendation for routine use of the SCORE algorithm in primary prevention of CVD is marked as class I. This means that there is "evidence and/or general agreement that a procedure is beneficial, useful, effective". Results of our overview indicate that in this case, class IIb ("usefulness/efficacy is less well established by evidence/opinion") would be more appropriate. In the British NICE clinical guideline CG181 [43] (concerning lipid modification for the primary and secondary prevention of CVD), the strength of the recommendations in individuals without CVD should also be lowered from "offer" to "consider". 
Researchers should plan and perform impact studies (probably RCTs) which take into account important endpoints e.g. CVD mortality and morbidity, to ultimately confirm the effectiveness or lack of effectiveness of TRA. Development of a new model of TRA and looking for the most appropriate format of its communication also seems inappropriate when effectiveness is not proven. Due to the probability of TRA ineffectiveness, the search for an utterly new method allowing the indication of points of onset and intensification of intervention in CVD primary prevention should be started.

Both researchers and guideline authors should be aware that it is inappropriate to use the models prepared and validated for prediction purposes to make clinical decisions concerning patients. It may be especially misleading if thresholds are based on opinions (which are cheap and quick) and not on impact studies.

Founders and policymakers ought to be aware that spending resources on adopting TRA into practice may be against current evidence which is not strong.

In the protocol of our overview, we expressed optimism that our study might allow patients to have information about a strong scientific background of performed CVD risk assessment. We had hoped that this knowledge would boost patients' adherence to their physicians' recommendations. However, these hopes have turned out to have been in vain.

\section{Conclusion}

SRs identified and included in our overview show a limited evidence, of low overall quality, suggesting a possible lack of effectiveness of TRA in reducing CVD events and mortality, as well as a clinically insignificant influence on individual risk factor levels. These studies also reveal that using TRA does not cause harm to patients. Presented results cannot be considered final and further research is still necessary, however broad implementation of TRA by practice might be devoid of justification based on evidence and should be reconsidered.

\section{Additional files}

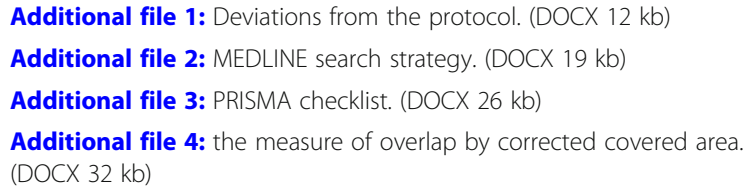

\section{Abbreviations}

ACC/AHA: American College of Cardiology/American Heart Association; BP: Blood pressure; CCA: Corrected cover area; Cl: Confidence interval; CVD: Cardiovascular disease; DBP: Diastolic blood pressure; HDL-C: High density lipoprotein; LDL-C: Low-density lipoprotein; MA: Meta-analysis; MD: Mean difference; NS: Non-significant; RCTs: Randomised control trials; RR: Risk ratio; SBP: Systolic blood pressure; SCORE: Systematic COronary Risk
Evaluation; SRs: Systematic reviews; TC: Total cholesterol; TRA: Total risk assessment; WHO: World Health Organization

\section{Acknowledgements}

Not applicable.

Funding

Publication was supported by the Jagiellonian University Medical College and the College of Family Physicians in Poland. The funding Organisations were not involved in the study design or protocol development.

\section{Availability of data and materials}

All data generated or analysed during this study are included in this published article [and its additional files].

\section{Authors' contributions}

$\mathrm{KS}$ and $\Pi \mathrm{T}$ conceived the study design. $\mathrm{KS}, \mathrm{T}, \mathrm{JK}, \mathrm{J}$, and $\mathrm{AW}$ contributed to developing the methods. KS, JK and TT developed the search strategy. JJ and AW provided methodological expertise. KS, TT and JK drafted the manuscript. KS, $T T, J K, J J$, and AW read, provided feedback on, and approved the final manuscript.

Ethics approval and consent to participate Not applicable.

\section{Consent for publication}

Not applicable.

\section{Competing interests}

All authors have completed the ICMJE uniform disclosure form at www.icmje.org/coi_disclosure.pdf and declare: no support from any organisation for the submitted work; TT reports personal fees from AbbVie Polska Sp. z o.o., personal fees from Eli Lilly Polska Sp. z o.o., non-financial support from VALEANT Polska Sp. z o.o., outside the submitted work. JJ reports personal fees from Valeant Polska sp. z o.o., personal fees from Mylan Polska sp. z o.o., non-financial support from $\mathrm{CHDE}$, non-financial support from Amgen Polska sp. z o.o. outside the submitted work. AW reports personal fees from MSD Polska sp. z 0.0., personal fees from Merck sp. z o.o., personal fees from Sanofi-Aventis sp. z o.o., personal fees from Polpharma SA, personal fees from Eli Lilly Polska sp. z o.o., personal fees from Valeant Polska sp. z o.o., outside the submitted work.

\section{Publisher's Note}

Springer Nature remains neutral with regard to jurisdictional claims in published maps and institutional affiliations.

\section{Author details}

${ }^{1}$ Department of Family Medicine, Chair of Internal Medicine and Gerontology, Jagiellonian University Medical College, 4 Bochenska Street, 31-061 Krakow, Poland. ²Department of Family Medicine and Public Health, Institute of Medicine, University of Opole, Opole, Poland. ${ }^{3}$ College of Family Physicians in Poland, Warszawa, Poland.

Received: 26 July 2018 Accepted: 21 December 2018

Published online: 09 January 2019

\section{References}

1. Lozano R, Naghavi M, Foreman K, Lim S, Shibuya K, Aboyans V, et al. Global and regional mortality from 235 causes of death for 20 age groups in 1990 and 2010: a systematic analysis for the global burden of disease study 2010. Lancet. 2012;380:2095-128. https://doi.org/10.1016/S0140-6736(12)61728-0.

2. Murray CJL, Barber RM, Foreman KJ, Ozgoren AA, Abd-Allah F, Abera SF, et al. Global, regional, and national disability-adjusted life years (DALYs) for 306 diseases and injuries and healthy life expectancy (HALE) for 188 countries, 1990-2013: quantifying the epidemiological transition. Lancet. 2015;386:2145-91. https://doi.org/10.1016/S0140-6736(15)61340-X.

3. Grundy SM, Pasternak R, Greenland P, Smith S, Fuster V. Assessment of cardiovascular risk by use of multiple-risk-factor assessment equations: a statement for healthcare professionals from the American Heart Association and the American College of Cardiology. J Am Coll Cardiol. 1999;34:1348-59.

4. Bundhun PK, Wu ZJ, Chen M-H. Impact of Modifiable Cardiovascular Risk Factors on Mortality After Percutaneous Coronary Intervention: A Systematic 
Review and Meta-Analysis of 100 Studies. Medicine (Baltimore). 2015;94: e2313. https://doi.org/10.1097/MD.0000000000002313.

5. KANNEL WB, DAWBER TR, KAGAN A, REVOTSKIE N, STOKES J. Factors of risk in the development of coronary heart disease--six year follow-up experience. The Framingham Study Ann Intern Med. 1961;55:33-50.

6. Piepoli MF, Hoes AW, Agewall S, Albus C, Brotons C, Catapano AL, et al. 2016 European guidelines on cardiovascular disease prevention in clinical practice. Eur Heart J. 2016;37:2315-81.

7. Giampaoli S, Palmieri L, Mattiello A, Panico S. Definition of high risk individuals to optimise strategies for primary prevention of cardiovascular diseases. Nutr Metab Cardiovasc Dis. 2005;15:79-85. https://doi.org/10.1016/j. numecd.2004.12.001.

8. Bitton A, Gaziano T. The Framingham heart Study's impact on global risk assessment. Prog Cardiovasc Dis. 2010;53:68-78. https://doi.org/10.1016/j. pcad.2010.04.001.

9. Damen JAAG, Hooft L, Schuit E, Debray TPA, Collins GS, Tzoulaki I, et al. Prediction models for cardiovascular disease risk in the general population: systematic review. Bmj. 2016:i2416. https://doi.org/10.1136/bmj.i2416.

10. Conroy RM, Pyörälä K, Fitzgerald AP, Sans S, Menotti A, De Backer G, et al. Estimation of ten-year risk of fatal cardiovascular disease in Europe: the SCORE project. Eur Heart J. 2003;24:987-1003.

11. Hippisley-Cox J, Coupland C, Vinogradova Y, Robson J, Minhas R, Sheikh A, et al. Predicting cardiovascular risk in England and Wales: prospective derivation and validation of QRISK2. BMJ. 2008;336:1475-82.

12. Goff DC, Lloyd-Jones DM, Bennett G, Coady S, D'Agostino RB, Gibbons R, et al. 2013 ACC/AHA guideline on the assessment of cardiovascular risk: a report of the American college of cardiology/American heart association task force on practice guidelines. Circulation. 2014;129(25 SUPPL. 1):S49-73.

13. Anderson TJ, Grégoire J, Hegele RA, Couture P, Mancini GBJ, McPherson R, et al. 2012 update of the Canadian cardiovascular society guidelines for the diagnosis and treatment of dyslipidemia for the prevention of cardiovascular disease in the adult. Can J Cardiol. 2013;29:151-67.

14. Stone NJ, Robinson JG, Lichtenstein AH, Bairey Merz CN, Blum CB, Eckel RH, et al. 2013 ACC/AHA guideline on the treatment of blood cholesterol to reduce atherosclerotic cardiovascular risk in adults: a report of the american college of cardiology/american heart association task force on practice guidelines. Circulation. 2014;129(25 SUPPL. 1):S1-45.

15. JBS 3 Board. Joint British Societies' consensus recommendations for the prevention of cardiovascular disease. Heart. 2014;100(Suppl):ii1-ii67.

16. Brindle P, Beswick A, Fahey T, Ebrahim S. Accuracy and impact of risk assessment in the primary prevention of cardiovascular disease: a systematic review. Heart. 2006;92:1752-9. https://doi.org/10.1136/hrt. 2006.087932.

17. Sheridan SL, Crespo E. Does the routine use of global coronary heart disease risk scores translate into clinical benefits or harms? A systematic review of the literature. BMC Health Serv Res. 2008;8:60. https://doi.org/ 10.1186/1472-6963-8-60

18. Sheridan SL, Viera AJ, Krantz MJ, Ice CL, Steinman LE, Peters KE, et al. The effect of giving global coronary risk information to adults: a systematic review - eAppendix. Arch Intern Med. 2010;170:e1-5.

19. Willis A, Davies M, Yates T, Khunti K. Primary prevention of cardiovascular disease using validated risk scores: a systematic review. J R Soc Med. 2012; 105:348-56. https://doi.org/10.1258/jrsm.2012.110193.

20. Studziński K, Tomasik T, Krzyszton J, Jóźwiak J, Windak A. Effect of using cardiovascular risk scoring in routine risk assessment in primary prevention of cardiovascular disease: protocol for an overview of systematic reviews. BMJ Open. 2017;7:e014206. https://doi.org/10.1136/bmjopen-2016-014206.

21. Montori VM, Wilczynski NL, Morgan D, Haynes RB. Optimal search strategies for retrieving systematic reviews from Medline: analytical survey. BMJ. 2005; 330:68.

22. Starr M, Chalmers I, Clarke M, Oxman AD. The origins, evolution, and future of the Cochrane database of systematic reviews. Int J Technol Assess Health Care. 2009;25(Suppl 1):182-95.

23. Moher D, Liberati A, Tetzlaff J, Altman DG. Preferred reporting items for systematic reviews and meta-analyses: the PRISMA statement. J Clin Epidemiol. 2009;62:1006-12.

24. Becker LA, Oxman AD. Overviews of reviews. In: Cochrane handbook for systematic reviews of interventions: Cochrane book series; 2008. p. 607-31.

25. Smith V, Devane D, Begley CM, Clarke M. Methodology in conducting a systematic review of systematic reviews of healthcare interventions. BMC Med Res Methodol. 2011;11:15. https://doi.org/10.1186/1471-2288-11-15.
26. Pieper D, Antoine S-L, Mathes T, Neugebauer EAM, Eikermann M. Systematic review finds overlapping reviews were not mentioned in every other overview. J Clin Epidemiol. 2014;67:368-75 http://www.embase.com/search/ results? subaction=viewrecord\&from $=$ export\&id $=L 372497994$.

27. Pieper $D$, Buechter $R$, Jerinic $P$, Eikermann $M$. Overviews of reviews often have limited rigor: a systematic review. J Clin Epidemiol. 2012;65:1267-73. https://doi.org/10.1016/j.jclinepi.2012.06.015.

28. Sharif MO, Janjua-Sharif FN, Sharif FNJ, Ali H, Ahmed F. Systematic reviews explained: AMSTAR-how to tell the good from the bad and the ugly. Oral Health Dent Manag. 2013;12:9-16 http//www.ncbi.nlm.nih.gov/pubmed/23474576.

29. Canadian Agency for Drugs and Technologies in Health (CADTH). Canadian Agency for Drugs and Technologies and Health. 2011. https://uww.cadth.ca/methodsdevelopment. Accessed 25 July 2018.

30. Ebrahim S, Taylor F, Ward K, Beswick A, Burke M, Davey SG. Multiple risk factor interventions for primary prevention of coronany heart disease. Cochrane Database Syst Rev. 2011:CD001561. https://doi.org/10.1002/14651858.CD001561.pub3.

31. Dyakova M, Shantikumar S, Colquitt JL, Drew CM, Sime M, Maclver J, et al. Systematic versus opportunistic risk assessment for the primary prevention of cardiovascular disease. Cochrane database Syst Rev. 2016:CD010411 https://www.cochranelibrary.com/cdsr/doi/10.1002/14651858.CD010411. pub2/abstract. Accessed 25 July 2018.

32. Krogsboll LT, Jorgensen KJ, Gronhoj Larsen C, Gotzsche PC, Krogsbøll LT, Jørgensen KJ, et al. General health checks in adults for reducing morbidity and mortality from disease: Cochrane systematic review and meta-analysis. BMJ. 2012;345(nov20 3):e7191. https://doi.org/10.1136/bmj.e7191.

33. Si S, Moss JR, Sullivan TR, Newton SS, Stocks NP. Effectiveness of general practice-based health checks: a systematic review and meta-analysis (Structured abstract). Br J Gen Pract. 2014;64:e47-53 https://www.ncbi.nlm. nih.gov/pmc/articles/PMC3876170/.

34. Ueda P, Woodward M, Lu Y, Hajifathalian K, Al-Wotayan R, Aguilar-Salinas CA, et al. Laboratory-based and office-based risk scores and charts to predict 10year risk of cardiovascular disease in 182 countries: a pooled analysis of prospective cohorts and health surveys. Lancet Diabetes Endocrinol. 2017. https://doi.org/10.1016/S2213-8587(17)30015-3.

35. Waldron C-A, van der Weijden T, Ludt S, Gallacher J, Elwyn G. What are effective strategies to communicate cardiovascular risk information to patients? A systematic review. Patient Educ Couns. 2011;82:169-81. https://doi.org/10.1016/j.pec.2010.04.014

36. van Dieren S, Beulens JWJ, Kengne AP, Peelen LM, Rutten GEHM, Woodward $M$, et al. Prediction models for the risk of cardiovascular disease in patients with type 2 diabetes: a systematic review. Heart. 2012;98:360-9.

37. Usher-Smith JA, Silarova B, Schuit E, Moons KGM, Griffin SJ. Impact of provision of cardiovascular disease risk estimates to healthcare professionals and patients: a systematic review. BMJ Open. 2015;5:e008717. https://doi.org/ 10.1136/bmjopen-2015-008717.

38. Tomasik T, Krzysztoń J, Dubas-Jakóbczyk K, Kijowska V, Windak A. The systematic coronary risk evaluation (SCORE) for the prevention of cardiovascular diseases. Does evidence exist for its effectiveness? A systematic review. Acta Cardiol. 2017; 72:1-10. https://doi.org/10.1080/00015385.2017.1335052.

39. Karmali KN, Persell SD, Perel P, Lloyd-Jones DM, Berendsen MA, Huffman MD, et al. Risk scoring for the primary prevention of cardiovascular disease. Cochrane Database Syst Rev. 2017. https://doi.org/10.1002/14651858.CD006887.pub3.

40. Collins DRJ, Tompson AC, Onakpoya IJ, Roberts N, Ward AM, Heneghan CJ. Global cardiovascular risk assessment in the primary prevention of cardiovascular disease in adults: systematic review of systematic reviews. BMJ Open. 2017;7:e013650. https://doi.org/10.1136/bmjopen-2016-013650.

41. MRFIT. Multiple risk factor intervention trial. Risk factor changes and mortality results. Multiple Risk Factor Intervention Trial Research Group. JAMA. 1982;248: 1465-77 http://www.ncbi.nlm.nih.gov/pubmed/7050440.

42. Kornitzer M, Rose G. WHO European collaborative trial of multifactorial prevention of coronary heart disease. Prev Med (Baltim). 1985;14:272-8.

43. National Institute for Health and Clinical Excellence. Lipid modification. Cardiovascular risk assessment and the modification of blood lipids for the primary and secondary prevention of cardiovascular disease. NICE clinical guideline CG181. 2014.

44. Jørgensen T, Jacobsen RK, Toft U, Aadahl M, Glümer C, Pisinger C. Effect of screening and lifestyle counselling on incidence of ischaemic heart disease in general population: Inter99 randomised trial. BMJ. 2014;348:93617. https://doi.org/10.1016/j.ypmed.2016.08.016.

45. Gotzsche PC, Jorgensen K, Krogsboll LT. General health checks don't work. BMJ. 2014;348(jun09 4):g3680. https://doi.org/10.1136/bmj.g3680. 